\title{
Eficiência do controle da ferrugem asiática da soja em função do momento de aplicação sob condições de epidemia em Londrina, PR
}

\author{
Cláudia V. Godoy ${ }^{1}$, Allan M. Flausino ${ }^{1}$, Leandro C.M. Santos ${ }^{2}$ \& Emerson M. Del Ponte ${ }^{3}$ \\ 'Embrapa Soja, 86001-970, Londrina, PR, Brasil; ${ }^{2}$ Faculdade Estadual de Filosofia, Ciências e Letras de Cornélio Procópio, \\ 86300-000, Cornélio Procópio, PR, Brasil; ${ }^{3}$ Departamento de Fitossanidade, Universidade Federal do Rio Grande do Sul, \\ 91540-000, Porto Alegre, RS, Brasil
}

Autor para correspondência: Cláudia V. Godoy, e-mail: godoy@cnpso.embrapa.br

\section{RESUMO}

Com o objetivo de estudar a eficiência do controle da ferrugem asiática da soja em função do momento de aplicação foram conduzidos ensaios em Londrina, PR, Brasil, durante as safras 2005/06 e 2006/07. A mistura de $60 \mathrm{~g}$ azoxistrobina ha ${ }^{-1}+24 \mathrm{~g}$ ciproconazol $\mathrm{ha}^{-1}$ foi aplicada em diferentes estádios fenológicos, iniciando em R2 até R5.5, em aplicações únicas e seqüenciais. A severidade foi estimada periodicamente para o cálculo da área abaixo da curva de progresso da doença (AACPD) e a produtividade avaliada no final do ciclo. Nas duas safras, os sintomas iniciais foram observados no final do estádio vegetativo. Na safra 2005/06, o tratamento com aplicações seqüenciais, em R2 e R5.1, foi o mais eficiente na redução da severidade e da AACPD e apresentou a maior produtividade. Na safra 2006/07, os tratamentos com aplicações seqüenciais, em R2 e R5.1, e a aplicação única em R3 apresentaram as menores severidades, menores AACPD e maiores produtividades. Correlações negativas foram encontradas entre as variáveis severidade em R6 e AACPD e a produtividade (-0,83 e -0,84 em 2005/06 e -0,87 e -0,89 em 2006/07). As aplicações realizadas com níveis elevados de severidade, ao redor de $50 \%$, apresentaram produtividade igual à testemunha não tratada.

Palavras-chave: Phakopsora pachyrhizi, Glycine Max, controle químico.

\begin{abstract}
Asian soybean rust control efficacy as a function of application timing under epidemic conditions in Londrina, PR

Fungicide trials were carried out in Londrina, PR, Brazil, during the 2005/06 and 2006/07 growing seasons with the objective of evaluating the efficacy of fungicide in Asian soybean rust control at different application times. A premix of $60 \mathrm{~g}^{2}$ azoxystrobin ha ${ }^{-1}$ $+24 \mathrm{~g}$ cyproconazole ha ${ }^{-1}$ was applied at different growth stages starting at R2 up to R5.5, and compared to two applications at R2 and R5.1. Following disease onset, disease severity was assessed periodically to calculate the area under disease progress curve (AUDPC). Plot yield was evaluated at harvest. Disease onset in both growing seasons occurred at the vegetative stage. In the 2005/06 season, the two-application treatment was the most efficient in reducing severity and AUDPC and increasing yield. In the 2006/07 season, both two applications and a single application at R3 reduced severity and AUDPC and increased yield compared to other treatments. A highly significant negative correlation was found between disease variables (severity at R6 and AUDPC) and yield in the two seasons (-0.83 and -0.84 in 2005/06 and -0.87 and -0.89 in 2006/07). Fungicide application with disease severity levels around $50 \%$ resulted in yield similar to the untreated control.
\end{abstract}

Keywords: Phakopsora pachyrhizi, Glycine max, chemical control.

A ferrugem Asiática da Soja (FAS), causada pelo fungo Phakopsora pachyrhizi Syd. \& P. Syd., é uma das doenças mais severas que incide na cultura da soja [Glycine $\max ($ L.) Merr.], com danos variando de $10 \%$ a $90 \%$ nas diversas regiões geográficas onde foi relatada (Sinclair \& Hartman, 1999; Yorinori et al., 2005). As primeiras epidemias severas de FAS na América do Sul foram relatadas no Paraguai, na safra 2000/01, e no Brasil, na safra 2001/02, nas regiões sul do estado de Goiás, no Mato Grosso, norte do Mato Grosso do Sul e no Rio Grande do Sul. (Yorinori et al., 2005). O custo da FAS no Brasil, desde as primeiras epidemias severas até a safra de 2007/08, foi estimado em aproximadamente US\$10,1 bilhões, incluindo as perdas em produção, arrecadação e o custo com o controle dessa doença (Consórcio Antiferrugem, 2008).

As condições climáticas exercem fundamental importância nas epidemias de FAS. O molhamento foliar contínuo, promovido por orvalho ou pela chuva, sob condições ótimas de temperatura $\left(18^{\circ} \mathrm{C}\right.$ a $\left.26,5^{\circ} \mathrm{C}\right)$ favorece o rápido desenvolvimento da doença (Melching et al., 1989; Alves et al., 2006), sendo a precipitação considerado o fator mais importante no progresso da doença nas condições de campo (Tschanz, 1984; Del Ponte et al., 2006). 
Para reduzir o risco de danos à cultura, as estratégias de manejo recomendadas no Brasil para essa doença são a utilização de cultivares de ciclo precoce e semeaduras no início da época recomendada; a eliminação de plantas de soja voluntárias e a ausência de cultivo de soja na entressafra; o monitoramento da lavoura desde o início do desenvolvimento da cultura e a utilização de fungicidas no aparecimento dos sintomas ou preventivamente (Tecnologias, 2006).

Estudos conduzidos no Brasil (Godoy, 2005a, 2005b; Godoy et al., 2007), Paraguai, África do Sul e Zimbábue (Miles et al., 2007) têm mostrado diferença de eficiência entre os produtos recomendados. No Brasil, os produtos registrados foram agrupados de acordo com análises de resultados de ensaios conduzidos sob alta pressão da doença, em diferentes regiões produtoras (Tecnologias, 2006). Com relação ao momento de aplicação, ensaios conduzidos em Zimbábue, entre 1998 e 2003, mostraram que três ou mais aplicações foram necessárias para manter o potencial produtivo, enquanto que uma ou duas aplicações, nas fases do enchimento de grãos, foram insuficientes para controlar a doença (Levy, 2005). Esses resultados sugeriram que o período crítico para proteção da cultura vai do estádio de florescimento (R1) até a maturação fisiológica (R7).

No Brasil, a média estimada do número de aplicações por hectare para controle da FAS na safra 2006/07 foi de 2,3 em uma safra em que as condições climáticas foram de maneira geral favoráveis às epidemias. No entanto, a cultura da soja nessa safra, atingiu a maior produtividade média histórica, de $2.822 \mathrm{~kg} \mathrm{ha}^{-1}$ (Conab, 2008), mostrando um eficiente controle da doença nas principais regiões produtoras. Aplicações de fungicida em soja realizadas de forma calendarizada, iniciando no estádio de florescimento e se repetindo em intervalos de 14 a 21 dias, podem promover uma adequada supressão da doença, embora ignore fatores que influenciam as epidemias, podendo resultar em aplicações desnecessárias. Esse tipo de situação é freqüentemente observado em vários outros patossistemas com intenso uso de fungicidas (Shtienberg, 2007).

Considerando a falta de estudos epidemiológicos detalhados acerca da eficiência do controle da FAS, em função do momento de aplicação, foram conduzidos ensaios em Londrina, PR, nas safras 2005/06 e 2006/07, com a cultivar BRS 154, considerada suscetível a FAS. Na safra 2005/06, a semeadura foi realizada em 19 de dezembro de 2005 e na safra 2006/07, em 11 de dezembro de 2006. As semeaduras foram realizadas no limite final da época recomendada com a finalidade de garantir maior quantidade de inóculo do fungo, proveniente das primeiras semeaduras na região.

A mistura pronta de $60 \mathrm{~g}$ azoxistrobina $\mathrm{ha}^{-1}+24$ g ciproconazol ha ${ }^{-1}$ com adjuvante Nimbus $5 \% \mathrm{v} / \mathrm{v}$ foi aplicada em diferentes estádios fenológicos, iniciando em R2 e finalizando em R5.5, em aplicações únicas e seqüenciais. As aplicações únicas foram realizadas em R2 (florescimento pleno), R3 (início de formação da vagem), R4 (vagem formada), R5.1 (início da formação das sementes), R5.3 (50\% da semente formada) e R5.5 (semente formada) (Fehr \& Caviness, 1981). Duas aplicações foram realizadas respectivamente em R2 eR5.1. O delineamento experimental foi em blocos ao acaso com quatro repetições, sendo cada repetição constituída de parcelas com seis linhas de largura, espaçadas de $45 \mathrm{~cm}$, e seis metros de comprimento. As quatro linhas centrais foram tratadas com fungicida e, as duas linhas centrais de cada parcela foram utilizadas para avaliação de produtividade. As aplicações foram realizadas utilizando pulverizador costal pressurizado com $\mathrm{CO}_{2}$ equipado com barra de dois metros com quatro bicos Teejet XR 11002, espaçados de $50 \mathrm{~cm}$ e calibrada para vazão de 200 litros hä ${ }^{-1}$.

A média de severidade das parcelas (área foliar coberta com sintomas) foi estimada semanalmente a partir da primeira aplicação com auxílio de escala diagramática (Godoy et al., 2006). Para estimativa da severidade média da parcela, foram avaliadas folhas nos terços inferior, médio e superior de quatro pontos na parcela. A área abaixo da curva de progresso da doença (AACPD) foi determinada pelo cálculo da integral da curva de progresso da doença, segundo Campbell \& Madden (1990).

A colheita foi realizada com colhedora de parcelas Wintersteiger modelo classic. A produtividade foi calculada em $\mathrm{kg} \mathrm{ha}^{-1}$, a $13 \%$ de umidade. Os tratamentos foram comparados por meio de análise de variância e teste de médias, empregando o software SASM-Agri (Canteri et al., 2001).

As aplicações iniciais, preconizadas nesse trabalho, foram realizadas seguindo a recomendação mais comumente utilizada no Brasil, com início no florescimento. No entanto, os primeiros sintomas, nas duas safras, foram observados no final do estádio vegetativo e, dessa forma, nenhum tratamento foi realizado preventivamente, ou seja, na ausência de sintomas. Na safra 2005/06, no momento da primeira aplicação em R2, a severidade média estimada foi $0,3 \%$ (Tabela 1) enquanto que, na safra 2006/07, a severidade foi de 1,2\% em R2 (Tabela 2). As severidades médias observadas nas parcelas testemunha, sem tratamento, foram de $65 \%$ e $85 \%$ em R6, para as safras 2005/06 e 2006/07, respectivamente (Tabelas 1 e 2).

$\mathrm{Na}$ safra 2005/06, o tratamento com aplicações seqüenciais realizadas em R2 e R5.1 foi o mais eficiente na redução da severidade e da AACPD, apresentando ainda a maior produtividade com diferença significativa em relação às aplicações únicas (Tabela 1). A segunda maior produtividade foi observada para o tratamento com aplicação em R4, com 13\% de severidade observada no momento da aplicação. O tratamento com aplicação em R3, apesar da menor severidade no momento da aplicação $(3,9 \%)$, apresentou produtividade inferior ao tratamento com aplicação em R4. Esse fato provavelmente é devido ao residual do produto não ter sido suficiente para proteção da cultura durante a fase de enchimento de grãos associado a distribuição de chuvas que favoreceu o progresso da doença 
TABELA 1 - Severidade da ferrugem da soja em R6 (\%), Área Abaixo da Curva de Progresso da Doença (AACPD), produtividade (kg $\mathrm{ha}^{-1}$ ) e redução de produtividade, em relação ao melhor tratamento (\%), dos diferentes tratamentos com a mistura de $60 \mathrm{~g}$ azoxistrobina ha-1 + 24 g ciproconazol ha ${ }^{-1}$ em aplicações únicas e seqüenciais em diferentes estádios fenológicos. Londrina, PR, safra 2005/06

\begin{tabular}{|c|c|c|c|c|c|c|}
\hline Tratamento $^{a}$ & $\begin{array}{c}\text { Severidade no } \\
\text { momento da aplicação } \\
(\%)\end{array}$ & $\begin{array}{c}\text { Severidade em } \\
\text { R6 (\%) }\end{array}$ & $\begin{array}{c}\text { Controle } \\
(\%)\end{array}$ & AACPD & $\begin{array}{c}\text { Produtividade } \\
\mathrm{kg} \mathrm{ha}^{-1}\end{array}$ & $\begin{array}{c}\text { Redução de } \\
\text { produtividade (\%) }\end{array}$ \\
\hline Testemunha & & $65 \mathrm{a}$ & & $1172 \mathrm{a}$ & $416 \mathrm{e}$ & 89 \\
\hline R3 & 3,9 & $19 \mathrm{~d}$ & 71 & $300 \mathrm{~d}$ & $1964 \mathrm{c}$ & 50 \\
\hline $\mathrm{R} 4$ & 13 & $14 \mathrm{~d}$ & 78 & $287 \mathrm{~d}$ & $2732 b$ & 30 \\
\hline $\mathrm{R} 5.1$ & 32 & $34 \mathrm{c}$ & 48 & $675 \mathrm{c}$ & $1057 \mathrm{~d}$ & 73 \\
\hline $\mathrm{R} 5.3$ & 52 & $53 \mathrm{~b}$ & 18 & $1022 \mathrm{~b}$ & $461 \mathrm{e}$ & 88 \\
\hline R5.5 & 65 & $58 \mathrm{~b}$ & 11 & $1096 \mathrm{~b}$ & $673 \mathrm{e}$ & 83 \\
\hline R2 e R5.1 & 0,3 & $7 \mathrm{e}$ & 89 & $149 \mathrm{e}$ & $3891 \mathrm{a}$ & 0 \\
\hline C.V. (\%) & & 14 & & 9 & 12 & \\
\hline
\end{tabular}

Médias seguidas pela mesma letra na vertical não diferem entre si pelo teste de Scott-Knott a 5\% de probabilidade.

${ }^{a}$ Estádio fenológico no momento das aplicações únicas ou seqüenciais.

TABELA 2 - Severidade da ferrugem da soja em R6 (\%), Área Abaixo da Curva de Progresso da Doença (AACPD), produtividade (kg $\mathrm{ha}^{-1}$ ) e redução de produtividade (\%), em relação ao melhor tratamento, dos diferentes tratamentos com a mistura de $60 \mathrm{~g}$ azoxistrobina ha ${ }^{-1}$ + 24 g ciproconazol ha ${ }^{-1}$ em aplicações únicas e seqüenciais em diferentes estádios fenológicos. Londrina, PR, safra 2006/07

\begin{tabular}{|c|c|c|c|c|c|c|}
\hline Tratamento $^{a}$ & $\begin{array}{c}\text { Severidade no } \\
\text { momento da aplicação } \\
(\%)\end{array}$ & $\begin{array}{l}\text { Severidade em } \\
\text { R6 }(\%)\end{array}$ & $\begin{array}{c}\text { Controle } \\
(\%)\end{array}$ & AACPD & $\begin{array}{l}\text { Produtividade } \\
\qquad \mathrm{kg} \mathrm{ha}^{-1}\end{array}$ & $\begin{array}{c}\text { Redução de } \\
\text { produtividade }(\%)\end{array}$ \\
\hline Testemunha & & $85 \mathrm{a}$ & & $936 a$ & $853 \mathrm{c}$ & 70 \\
\hline $\mathrm{R} 2$ & 1,2 & $55 \mathrm{~b}$ & 35 & $346 \mathrm{c}$ & $1731 \mathrm{~b}$ & 38 \\
\hline R3 & 2 & $12 \mathrm{~d}$ & 86 & $160 \mathrm{~d}$ & $2306 \mathrm{a}$ & 18 \\
\hline $\mathrm{R} 4$ & 2,5 & $26 \mathrm{c}$ & 69 & $345 \mathrm{c}$ & $1875 \mathrm{~b}$ & 33 \\
\hline R5.1 & 12 & $58 \mathrm{~b}$ & 32 & $555 \mathrm{~b}$ & $1587 \mathrm{~b}$ & 43 \\
\hline R5.3 & 48 & $77 \mathrm{a}$ & 9 & $876 \mathrm{a}$ & $855 \mathrm{c}$ & 70 \\
\hline $\mathrm{R} 5.5$ & 60 & $81 \mathrm{a}$ & 5 & $891 \mathrm{a}$ & $852 \mathrm{c}$ & 70 \\
\hline R2 and R5.1 & 1,2 & $7 \mathrm{~d}$ & 92 & $140 \mathrm{~d}$ & $2808 \mathrm{a}$ & 0 \\
\hline C.V.(\%) & & 20 & & 12 & 14 & \\
\hline
\end{tabular}

Médias seguidas pela mesma letra na vertical não diferem entre si pelo teste de Scott-Knott a 5\% de probabilidade.

${ }^{a}$ Estádio fenológico no momento das aplicações únicas ou seqüenciais.

(Figura 1A). Nessa safra, o tratamento com aplicação única em R2 foi perdido devido a problemas na condução do ensaio. As correlações entre as variáveis de doença e produtividade foram elevadas, sendo de $-0,83(p<0,01)$ e $-0,84(p<0,01)$ para severidade em R6 e AACPD, respectivamente.

$\mathrm{Na}$ safra 2006/07, os tratamentos com duas aplicações realizadas em R2 e R5.1 e a aplicação única em R3 apresentaram as menores severidades finais, menores AACPD e maiores produtividades, comparados aos demais tratamentos (Tabela 2). A aplicação em R2, com menor nível de severidade, apresentou severidade final elevada (55\%), sendo a produtividade média inferior ao tratamento com duas aplicações, também devido ao residual não ter sido suficiente para proteção da cultura durante a fase de enchimento de grãos. A produtividade para o tratamento com aplicação em R2 foi estatisticamente semelhante aos tratamentos com aplicações em R4 e R5. De forma semelhante à safra 2005/06, as correlações entre as variáveis de doença e a produtividade foram elevadas, sendo de $-0,87$ $(p<0,01)$ e $-0,89(p<0,01)$ para severidade em R6 e AACPD, respectivamente.

$\mathrm{Na}$ safra 2005/06, o progresso da ferrugem foi mais rápido, principalmente entre os períodos de $\mathrm{R} 2 \mathrm{e}$ R5 (Tabela 1 e Figura 1A), quando comparado com o progresso na safra 2006/07 (Tabela 2 e Figura 1B), onde a severidade em R2 era maior. Isso pode ser explicado pela distribuição de chuvas que ocorreu nos dois anos (Figura 1). Em 2005/06, a quantidade de chuva entre R2 e R5 foi de $269 \mathrm{~mm}$ distribuídos em 15 dos 22 dias entre os dois estádios fenológicos. Em 2006/07, o acumulado de chuva nesse mesmo período foi de $138 \mathrm{~mm}$ em 12 dos 25 dias entre os dois estádios fenológicos. A relação entre as condições de chuva e a severidade final nas epidemias foi demonstrada por Del Ponte et al. (2006), sendo confirmada 

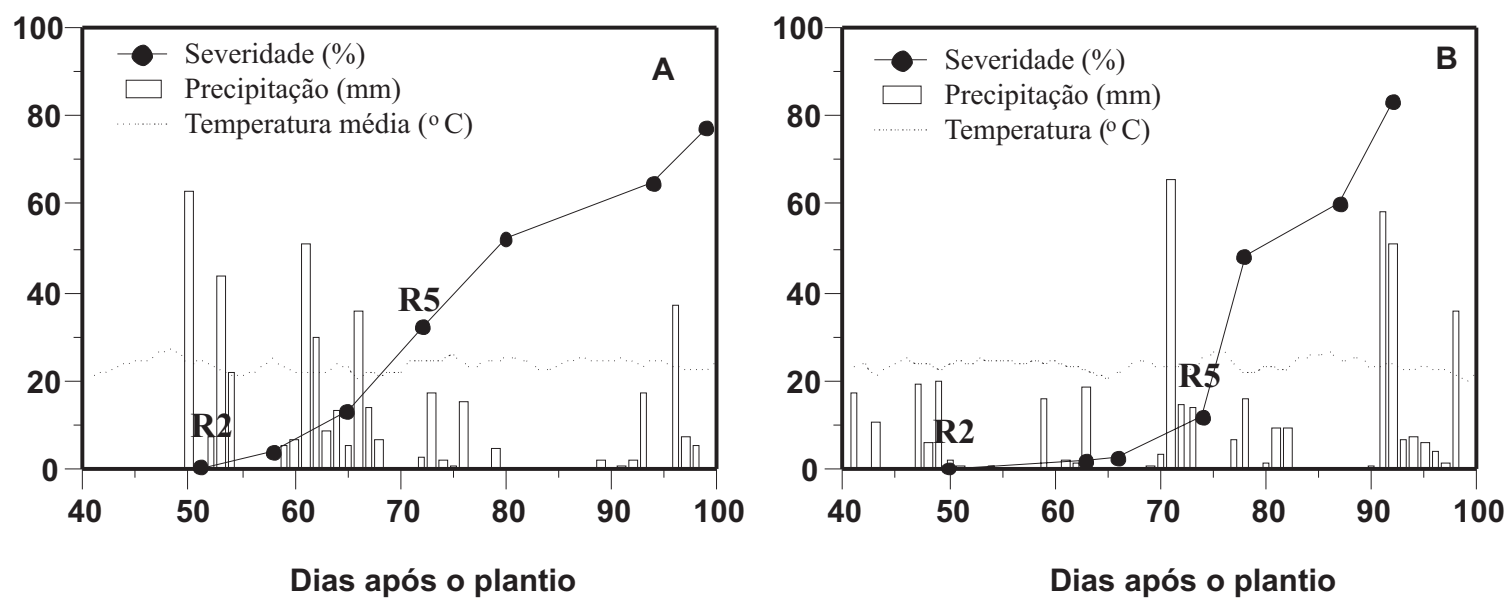

FIGURA 1 - Curvas de progresso da ferrugem no tratamento sem fungicida, precipitação diária $(\mathrm{mm})$ e temperatura média $\left({ }^{\circ} \mathrm{C}\right)$, em Londrina, PR, Brasil, nas safras 2005/06 (A) e 2006/07 (B).

nesses ensaios. A maior freqüência de chuvas na safra 2005/06 influenciou o período residual do fungicida e conseqüentemente, a eficiência de controle, uma vez que nessa safra nenhuma aplicação isolada apresentou controle semelhante ao tratamento com duas aplicações.

$\mathrm{Na}$ safra 2006/07, devido a menor freqüência de chuva comparada à safra anterior, a ferrugem progrediu mais lentamente depois de constatados os sintomas iniciais e o tratamento com aplicação única no estádio R3 apresentou controle semelhante a duas aplicações. O tratamento realizado em $\mathrm{R} 4$, apesar de valores semelhantes de severidade, apresentou severidade final superior e menor produtividade. Uma das hipóteses para essa menor eficiência de controle pode ser devido ao índice de área foliar (IAF) no momento da aplicação. Apesar da variedade apresentar hábito de crescimento determinado, o IAF continua a aumentar até o estádio R5 (Carpenter \& Board, 1997; Kumudini et al., 2008) e, dessa forma, a penetração dos fungicidas pode não ter proporcionado cobertura tão eficiente como a aplicação realizada em R3.

Embora trabalhos realizados em Zimbábue tenham comprovado a necessidade de três aplicações em condições de alta severidade da doença (Levy, 2005), esse fato não foi observado nesses ensaios. Mesmo com aplicações iniciando após a constatação dos sintomas iniciais, a eficiência de controle, quando comparada à severidade da testemunha sem controle e dos melhores tratamentos, foi de $89 \%$ na safra 2005/06 (Tabela 1), para o tratamento com duas aplicações, e de $92 \%$ e $86 \%$ na safra 2006/07, para os tratamentos com duas aplicações e aplicação única em R3 (Tabela 2), respectivamente. Uma das razões para a diferença observada nesse trabalho em relação ao trabalho em Zimbábue pode ter ocorrido devido aos diferentes princípios ativos dos fungicidas utilizados. $\mathrm{O}$ produto utilizado nos ensaios no Zimbábue foi a mistura pronta de carbendazim + fluzilazol, que nos ensaios em rede, realizados em situações de epidemia na safra 2004/05, foi inferior a mistura azoxistrobina + ciproconazol, utilizada nesse trabalho (Godoy, 2005b). É importante salientar que aplicações calendarizadas em R2 e em R5 nem sempre garantem uma boa eficiência de controle, conforme observado nos ensaios em rede realizados na safra 2006/07, conduzidos sob alta pressão da doença, onde a média da eficiência máxima observada com aplicações em R2 e R5 foi de 70\% (Godoy et al., 2007).

Os resultados obtidos nesse trabalho reforçam a importância do monitoramento da doença na tomada de decisão para o início das aplicações e o monitoramento da lavoura e do ambiente para a determinação do intervalo entre aplicações. O momento da primeira aplicação é um dos fatores mais críticos para o sucesso do controle e atrasos significativos na aplicação, após o estabelecimento da doença em níveis elevados, podem resultar em perdas significativas de produtividade (Levy, 2005; Miles et al., 2004).

As aplicações realizadas com níveis elevados de severidade, de $52 \%$ e $48 \%$ nas safras $2005 / 06$ e $2006 / 07$, não mostraram retorno em produtividade, sendo as reduções médias de produtividade de $86 \%$ e de $70 \%$, respectivamente, semelhantes à testemunha sem controle. Esse mesmo fato foi observado por Navarro et al. (2004) onde a produtividade de plantas tratadas com elevada severidade se mostrou semelhante à produtividade de plantas sem controle. Por outro lado, produtividades elevadas foram observadas no presente trabalho com aplicações isoladas ou seqüenciais após o estabelecimento da doença, em níveis de até $2 \%$ de severidade. As razões para os produtores no Brasil, e de outros lugares do mundo, adotarem programas de aplicações calendarizadas têm base na dificuldade de identificar a doença no seu início e o potencial de dano sob situações de falha no manejo. Em Zimbábue e na África do Sul as recomendações de controle também são feitas seguindo 
o esquema calendarizado, a partir do florescimento e se repetindo em intervalos de 21 dias, sendo recomendadas três aplicações em áreas com alta intensidade da doença e duas em áreas com baixa intensidade (Levy, 2005).

Os resultados aqui apresentados são de ensaios conduzidos em situação de alto nível de inóculo e ambiente favorável à infecção inicial, uma vez que os primeiros sintomas foram observados ainda no estádio vegetativo. De acordo com observações realizadas após a constatação da FAS no Brasil esse fato ocorre mais comumente em semeaduras tardias em relação à época recomendada. Recentemente a incidência da FAS no período vegetativo tem sido reduzida com as recomendações de semeadura no início da época recomendada, e com a redução do inóculo na entressafra devido à adoção do "vazio sanitário" a partir de 2006, em alguns Estados do Brasil, o qual consiste em um período de 60 a 90 dias sem soja na entressafra. Considerando a variação nos padrões da doença nas diferentes regiões do Brasil, devido à influência do inóculo e das condições climáticas, variáveis a cada safra, não se justifica a adoção de um modelo único para o manejo da doença. É importante que esse seja feito de forma racional em função da situação local, onde o uso de fungicidas deve ser planejado de acordo com os fatores de risco que são monitorados no decorrer da safra, e a época de início das aplicações e reaplicações no momento correto são de importância fundamental no controle da doença e garantia de maiores lucros aos produtores.

\section{AGRADECIMENTOS}

Manuscrito aprovado para publicação pelo Comitê de Publicações da Embrapa Soja sob o número 03/2008.

\section{REFERÊNCIAS BIBLIOGRÁFICAS}

Alves SAM, Furtado GQ, Bergamin Filho, A (2006) Influência das condições climáticas sobre a ferrugem da soja. In: Zambolim L (Ed.). Ferrugem asiática da soja. Viçosa MG. Suprema Gráfica e Editora Ltda. pp. 37-59.

Campbell CL, Madden, LV (1990) Introduction to plant disease epidemiology. New York NY. John Willey \& Sons.

Canteri MG, Althaus RA, Virgens Filho JS, Giglioti EA, Godoy CV (2001) SASM-Agri - Sistema para análise e separação de médias em experimentos agrícolas pelos métodos Scott-Knott, Tukey e Duncan. Revista Brasileira de Agrocomputação 1:18-24.

Carpenter AC, Board JE (1997) Growth dynamic factors controlling soybean yield stability across plant populations. Crop Science 37:1520-1526.

CONAB. Série histórica. www.conab.gov.br/conabweb/download/ safra/SojaSerieHist.xls

CONSÓRCIO ANTIFERRUGEM. Conheça a ferrugem: Tabela de custo. www.consorcioantiferrugem.net/index.php?download=tabela custos_ferrugem $\% 202008$.pdf

Del Ponte EM, Godoy CV, Li X, Yang XB (2006) Predicting severity of Asian soybean rust epidemics with empirical rainfall models. Phytopathology 96:797-803.

Fehr WR, Caviness CE (1981) Stages of soybean development. Iowa State University. Special Report 80.

Godoy CV (2005a) Resultados da rede de ensaios para controle químico de doenças na cultura da soja. Safra 2003/2004. Londrina PR. Embrapa Soja (Embrapa Soja. Documentos 251).

Godoy CV (2005b) Ensaios em rede para controle de doenças na cultura da soja. Safra 2004/2005. Londrina: Embrapa Soja (Embrapa Soja. Documentos 266).

Godoy CV, Koga LJ, Canteri MG (2006) Diagrammatic scale for assessment of soybean rust severity. Fitopatologia Brasileira 31:63-68.

Godoy CV, Pimenta CB, Miguel-Wruck DS, Ramos Junior EU, Siqueri FV, Feksa HR, Dos Santos I, Lopes ION, Nunes Junior J, Ito MA, Iamamoto MM, Ito MF, Meyer MC, Dias M, Martins MC, Almeida NS, Andrade NS, Andrade PJM, Sourza PIM, Balardin RS, Barros R, Silva SA, Furlan SH, Gavassoni WL (2007) Eficiência de fungicidas para controle da ferrugem asiática da soja, Phakopsora pachyrhizi, na safra 2006/07. Resultados sumarizados dos ensaios em rede. Londrina: Embrapa Soja. (Embrapa Soja. Circular Técnica 42).

Kumudini S, Godoy CV, Board JE, Omielan J, Tollenaar M (2008). Mechanisms involved in soybean rust-induced yield reduction. Crop Science 48:2334-2342.

Levy C (2005) Epidemiology and chemical control of soybean rust in southern Africa. Plant Disease 89: 669-674.

Melching JS, Dowler WM, Koogle DL, Royer MH (1989) Effect of duration, frequency, and temperature of leaf wetness period on soybean rust. Plant Disease 73:117-122.

Miles MR, Levy C, Hartman GL (2004) Summary of the USDA fungicide efficacy trials to control soybean rust in Zimbabwe 20032004. Integrated Pest Management Reviews. www.ipmcenters. org/NewsAlerts/soybeanrust/Zimbabwe2004EfficacyTrials.pdf

Miles MR, Levy C, Morel W, Mueller T, Steinlage T, Van Rij N, Frederick RD, Hartman GL (2007) International fungicide efficacy trials for the management of soybean rust. Plant Disease 91:1450-1458.

Navarro JC, Nakasato R, Utiamada CM, Yorinori JT (2004) First report $\mathrm{f}$ Asian soybean rust in Bolivia. Proceedings VII World Soybean Research Conference, IV International Soybean Processing and Utilization Conference, III Congresso Brasileiro de Soja (Brazilian Soybean Congress), Foz do Iguaçu, PR. pp. 8586.

Shtienberg D (2007) The contribution of epidemiological research to plant disease management. Australasian Plant Pathology 36:510-515.

Sinclair JB, Hartaman GL (1999) Soybean rust. In: Hartman GL, Sinclair JB, Rupe JC (Eds.). Compendium of soybean diseases. 4. ed. Saint Paul MN. APS Press. pp. 25-26.

Tecnologias de produção de soja - região central do Brasil 2007. (2006) Londrina PR. Embrapa Soja. Embrapa Cerrados. Embrapa Agropecuária Oeste. 
Eficiência do controle da ferrugem asiática da soja em função do momento...

Tschanz AT (1984) Soybean rust epidemiology: Final Report. Asian Vegetable Research and Development Center, Shanhua, Taiwan.
Yorinori JT, Paiva WM, Frederick RD, Costamilan LM, Bertagnolli PF, Hartman GE, Godoy CV, Nunes Junior J (2005) Epidemics of soybean rust (Phakopsora pachyrhizi) in Brazil and Paraguay from 2001 to 2003. Plant Disease 89:675-677.

Recebido 4 Março 2008 - Aceito 3 Fevereiro 2009 - TPP 8023

Editor Associado: David S. Jaccoud Filho 